\title{
Shape or friction? Which of these characteristics drives the shear strength in granular systems?
}

\author{
Theechalit Binaree ${ }^{1}$, Emilien Azéma ${ }^{2,3}$, Nicolas Estrada ${ }^{4}$, Mathieu Renouf $^{2}$, and Itthichai Preechawuttipong ${ }^{1, *}$ \\ ${ }^{1}$ Department of Mechanical Engineering, Chiang Mai University, Chiang Mai, Thailand \\ ${ }^{2}$ LMGC, Université de Montpellier, CNRS, Montpellier, France \\ ${ }^{3}$ Institut Universitaire de France (IUF), Paris, France \\ ${ }^{4}$ Departamento de Ingeniería Civil y Ambiental, Universidad de Los Andes, Bogotá, Colombia
}

\begin{abstract}
The shape of the particles and local friction, separately, are known to strongly affect the macroscopic properties of an assembly of grains. But the combined effects of these two parameters still remain poorly described. By means of extensive two dimensional contact dynamics simulations, we perform a systematic analysis of the interplay between friction and shape on strength properties of granular systems. The shape of the particles is varied from disks to triangles, while the friction is varied from 0 to 0.7 . We find that the macroscopic friction first increases with angularity, but it may decline (for low friction values), saturate (for intermediates friction values), or continue to increase (for large friction values) for the most angular shapes. In other words, the effect of the particle's angularity on the shear strength depends on the level of sliding friction. In contrast, the effect of local friction on the shear strength does not depend on the specific properties of shape. The results presented here highlight the subtle coupling existing between shape and friction effects.
\end{abstract}

\section{Introduction}

Particle shape is an intrinsic characteristic that plays an important role in the microstructure and mechanical behavior of granular media [1]. Several studies have recently been carried out for angular shapes [2-7], elongated particles [8-11], non-convex shapes [12], as well as for "super-balls" [13]. All these studies have revealed rather unexpected behaviors, such as the non-linear increase of shear strength as particle's angularity is increased [4] or the non linear variation of the packing fraction with respect to the particle's aspect ratio [9], to name a few. The non-linear variation in strength finds its origin in subtle shape-dependent compensation mechanisms at the microscopic scale between stress transmission, the mobilization of friction, and grain connectivity.

Along with particle shape, local friction is a key element in the build-up of anisotropic structures at the origins of strength. Basically, it has been constantly shown in the literature that shear strength first increases with local friction and then saturates at a constant value, even if local friction continues to increase [14-20]. This has been shown in assemblies of disks, spheres, and cube-like particles [21, 22]. These results have been verified in a parametric study where the particles interact by means of both sliding friction and high rolling resistance $[18,23]$. But the use of a rolling resistance in disks assemblies ignores the geometrical restrictions induced by a non-circular shape. In this sense, such studies assume that shape and friction are uncoupled.

*e-mail: itthichai.p@cmu.ac.th

A video is available at https://doi.org/10.48448/z8qa-kq03
The aim of this work is thus to take a step forward in the understanding of the joint effect of local variables by considering the effects of friction and particle shape on the macroscopic strength of granular systems. In the following, we present the numerical procedures to build and test the shear strength of samples composed of (2D) angular grains (Sec. 2). In Sec. 3, we focus on the evolution of shear strength and packing fraction as a function of shape and friction. We conclude in Sec. 4, with a summary and some perspectives.

\section{Numerical setup}

We use the Contact Dynamics (CD) method, a class of discrete element method (DEM), with irregular polygonal particles [24, 25]. The CD method is based on implicit time integration of the equations of motion together with a nonsmooth formulation (i.e no regularization) of the contact normal force and dry friction. Consequently, the only physical parameter is the coefficient of friction $\mu_{s}$, which is varied in the set $\{0,0.01,0.05,0.1,0.2,0.3,0.4,0.5,0.6,0.7\}$. The method is implemented in the LMGC90 plateforme ${ }^{1}$, a multipurpose software developed in Montpellier, capable of modeling collections of deformable or undeformable particles of various shapes by means of different algorithms as well as a parallel version to ensure reasonable CPU times [26].

Each numerical sample is composed of 10000 particles, geometrically deposed and randomly oriented in a

\footnotetext{
https://git-xen.lmgc.univ-montp2.fr/lmgc90/lmgc90_user/wikis/home
} 
rectangular box with frictonnless walls. We prepare 11 packings, each of them composed of particles having the same number of sides $n_{s}$ : one packing of disks and 10 packings with $n_{s} \in[50,30,20,10,8,7,6,5,4,3]$. In addition, shape properties were described by the angularity, $\alpha=2 \pi / n_{s}$. In order to avoid local ordering, a slight polydispersity in size and shape is introduced. Particle size is defined from the circumscribed circle diameter of each polygon and varied between $d_{\min }$ and $d_{\max }$, where $d_{\max }=1.25 d_{\min }$, with an uniform distribution per volume (area) fraction. Particle shape polydispersity is controlled by the level of irregularity of the polygons by randomly perturbing the position of the vertices over a small interval angle. In all cases, gravity and friction between particles and walls are set to zero.

For each combination of $n_{s}$ and $\mu_{s}$, the packing is isotropically compressed under a stress $\sigma_{0}$ applied at the top and right walls, while the left and bottom walls are kept fixed. At the end of isotropic compression, this is when a jammed mechanically stable configuration of particles is obtained (see the example in Fig. 1), a bi-axial compression is imposed. We impose a vertical compression with a constant velocity $v_{0}$ and a lateral constant confining stress $\sigma_{0}$. Quasi-static limit behavior is considered in this study, controlled through the inertial number $I$, set to $5 \times 10^{-4}$.

\section{Numerical result}

\subsection{Definition of macroscopic quantities}

For a $2 \mathrm{D}$ system, we define the deviatoric stress as $q=$ $\left(\sigma_{1}-\sigma_{2}\right) / 2$ and the mean stress as $p=\left(\sigma_{1}+\sigma_{2}\right) / 2$, with $\sigma_{1}$ and $\sigma_{2}$ being the principal values of the stress tensor $\sigma$. In a granular system, $\sigma$ is defined from the contact scale by the well-known formula [27]:

$$
\sigma_{i j}=\frac{1}{V} \sum_{c \in V} f_{i}^{c} l_{i}^{c},
$$

where $\boldsymbol{f}$ is the contact force, $\boldsymbol{\ell}$ is the branch vector (i.e., the vector joining the centers of particles interacting at contact $c$ ) and the sum runs over all contacts in a control volume $V$. According to the Mohr-Coulomb model, the shear strength is defined from the ratio [1]:

$$
\mu=\frac{q}{p} .
$$

We also define the vertical strain $\varepsilon_{1}=\Delta h / h_{0}$, where $h$ and $h_{0}$ are the height and initial height of the box, respectively, and $\Delta h=h_{0}-h$ is the cumulative displacement. Finally, the packing fraction is given by:

$$
\rho=\frac{\sum_{p} V_{p}}{V}
$$

where $V_{p}$ is the volume of a particle $p$ inside the volume of the box $V$.

\subsection{Stress-strain behavior and effects of local parameters}

Figure 2 shows the evolution of $q / p$ and $\rho$ (inset) as functions of the vertical strain $\varepsilon_{1}$ : (a) for packings made of

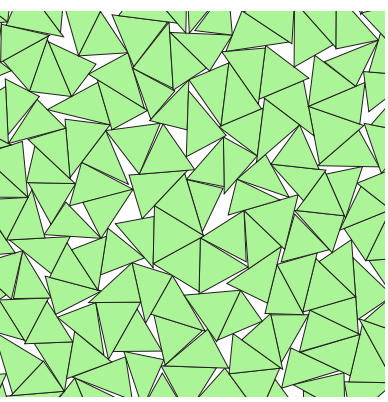

(a)

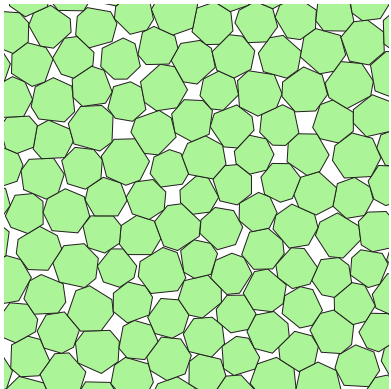

(c)

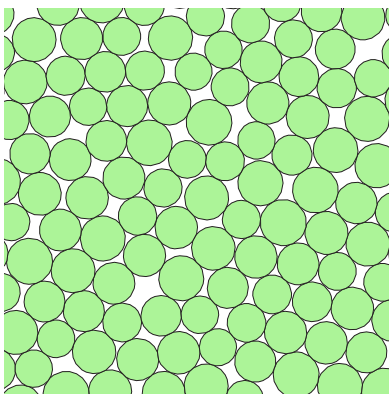

(e)

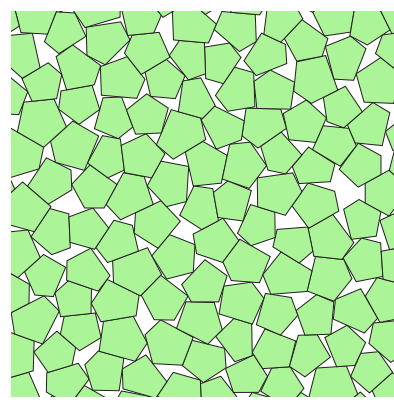

(b)

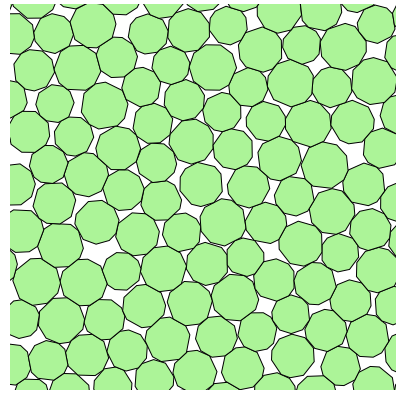

(d)

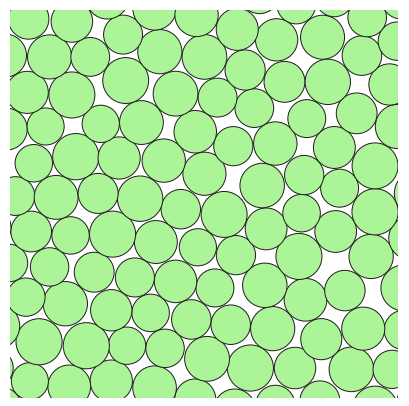

(f)
Figure 1. Particle scale views of dense samples at end isotropic compression: (a) $n_{s}=3$, (b) $n_{s}=5$, (c) $n_{s}=7$, (d) $n_{s}=10$,(e) $n_{s}=20$, and (f) disks.

pentagons and for all values of $\mu_{s}$, and (b) for $\mu_{s}=0.5$ and all shapes simulated in this paper. Basically, for all frictions and shapes the shear strength first increases from 0 and then reaches a constant shape-dependent and frictiondependent value for larger deformations. Typically, for $\varepsilon_{1}>0.2$ we can consider that the shear strength is constant up to small fluctuations. But what is also interesting to note on these figures is that there is a stress peak $(q / p$ goes through a maximum) only if the inter-granular friction is greater than $\mu_{s}>0.1$. Let us recall that, due to isotropic compression, all samples are initially dense, and thus the existence of the strength peak cannot be attributed exclusively to high values of the packing fraction.

Along the same line, we see that the packing fraction $\rho$ decreases from its initial value and tends toward a constant value, also for $\varepsilon_{1}>0.2$. From these observations, we thus define the residual shear strength $\mu^{*}$ and the residual packing fraction $\rho^{*}$ as the mean values of $\mu$ ( $\rho$ respectively) in the range of $\varepsilon_{1} \in[0.2,0.4]$.

The variations of the residual shear strength $\mu^{*}$ as a function of friction $\mu_{s}$ and for all angularities $\alpha$ are shown 


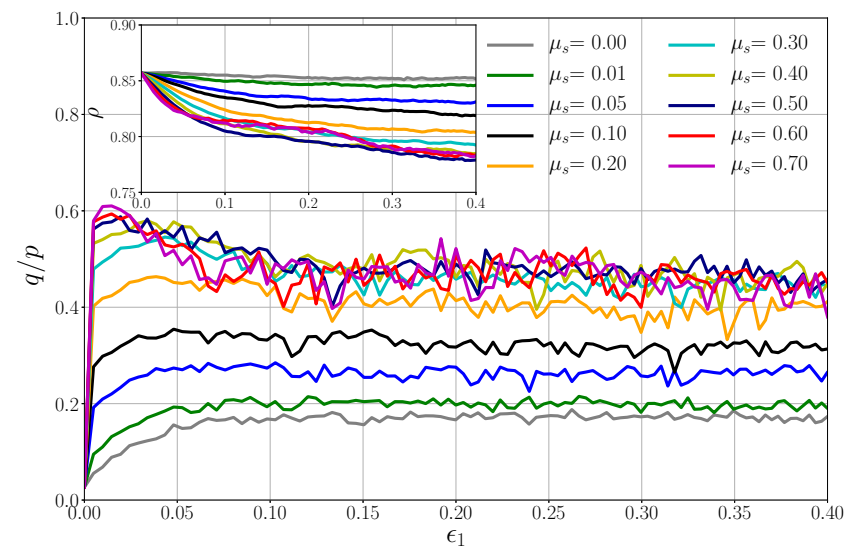

(a)

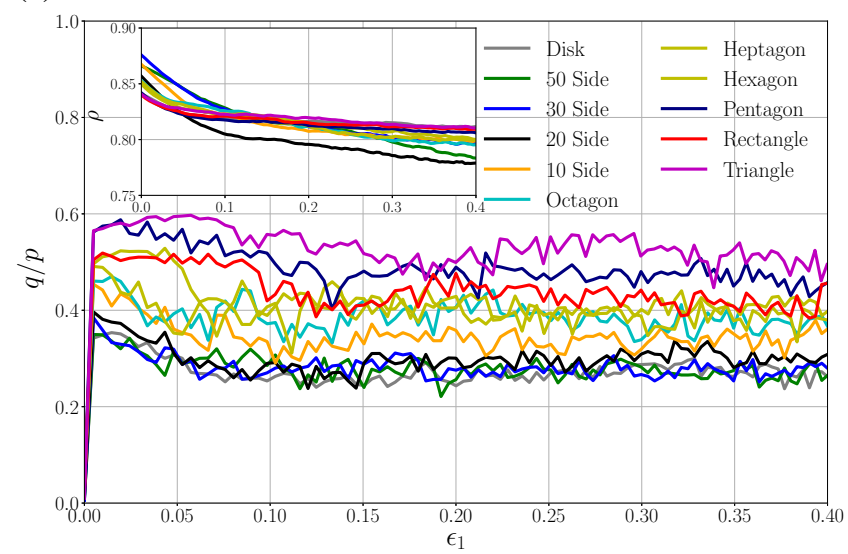

(b)

Figure 2. Stress ratio $q / p$ and solid fraction $\rho$ (inset) as functions of the vertical strain $\varepsilon_{1}$. (a) Packings of pentagons for all values of the local friction $\mu_{s}$. (b) Packings with $\mu_{s}=0.5$ for all shapes.

in Fig. 3, and as a function of $\alpha$ for all $\mu_{s}$ in Fig. 4. Basically, for all shapes a similar trend is observed. This is, $\mu^{*}$ first increases with $\mu_{s}$ and then saturates at a shapedependent value. Our result is consistent with previous works with assemblies of disks, spheres, or cube-like particles in 3D [16, 18, 20, 21, 28].

In contrast, the evolution of $\mu^{*}$ with $\alpha$ clearly depends on $\mu_{s}$. For weakly frictional systems (i.e., for $\mu_{s}$ below $0.3), \mu^{*}$ first increases with $\alpha$ but it declines as particles become more angular (i.e., for $n_{s}$ below 6). This means that a weakly frictional packing composed of triangles or squares can have a macroscopic friction that is smaller than that of a packing of disks. For intermediate values of friction, typically for $\mu_{s} \in[0.3,0.4], \mu^{*}$ remains constant, while it continues to increase for frictions larger than 0.4 . This behavior is rather unexpected and clearly suggests that representing particle shape through a rolling friction coefficient in circular particle assemblies may be acceptable for intermediate values of sliding friction [29], but it is not sufficient for small, or on the contrary large, friction values.

Finally, in the insets of Figs. 3 and 4 we show the variations of the residual packing fraction $\rho^{*}$ as a function

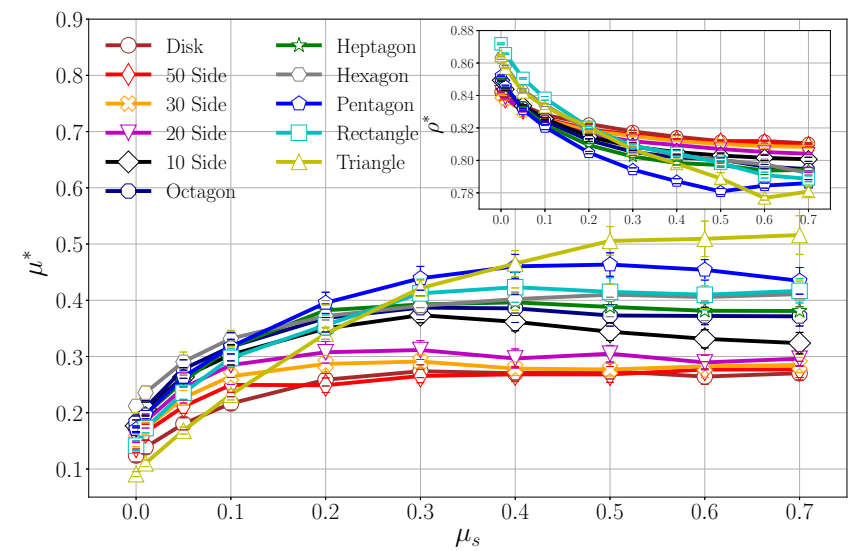

Figure 3. Macroscopic friction coefficient $\mu^{*}$ and packing fraction $\rho^{*}$ (inset) in the steady state, as functions of the contact friction $\mu_{s}$. The error bars show the standard deviation in the steady state.

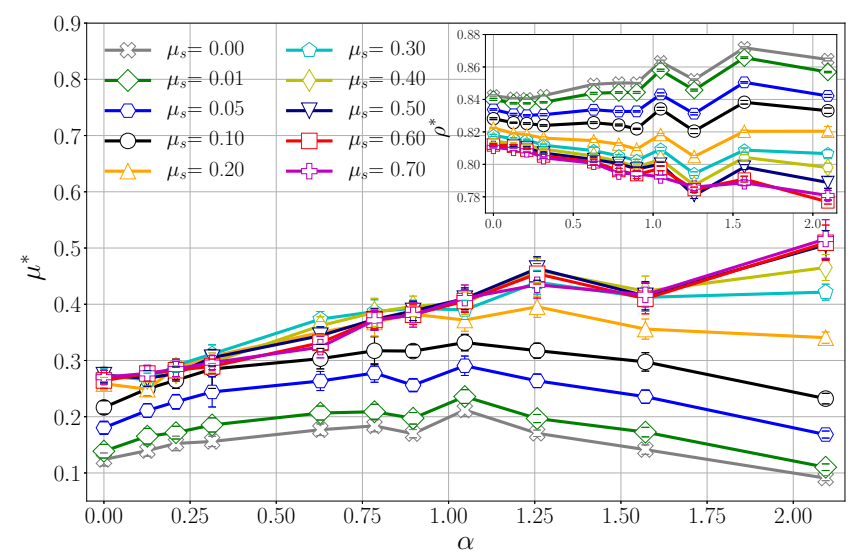

Figure 4. Macroscopic friction coefficient $\mu^{*}$ and packing fraction $\rho^{*}$ (inset) in the steady state, as functions of the particle angularity $\alpha$. The error bars show the standard deviation in the steady state.

of $\mu_{s}$ for all $\alpha$, and as a function of $\alpha$ for all $\mu_{s}$, respectively. Basically, $\rho^{*}$ varies conversely as $\mu^{*}$. More precisely, $\rho^{*}$ increases with $\alpha$ in weakly frictional systems and declines with $\alpha$ for the largest values of $\mu_{s}$.

\section{Conclusions}

To conclude, by means of two dimensional Contact Dynamics simulations, we presented a systematic analysis of the coupled effects of particle shape and local friction on the strength properties of granular systems. Assemblies composed of irregular polygonal particles were systematically sheared from their initial state to a large deformation until a steady state was reached. The shape of the particles was varied from triangles to disks and friction was varied from 0 to 0.7 .

For all shapes, we find that the shear strength first increases with local friction and then saturates at a shapedependent value. This result extends previous results ob- 
tained for simplified shapes to a large variety of shapes. But, a counterintuitive finding of this work is that the effect of particle shape on the shear strength strongly depends on the value of the local friction. The shear strength first increases for all shapes, but it may decline, saturate or continue to increase at larger angularity depending on the value of the local friction. This behavior is rather unexpected, and suggests a complex interplay at the microscopic scale [30]. Furthermore, our numerical results clearly highlight that attempting to represent noncircular/non-spherical shapes by hindering the rotations in assemblies of circular/spherical particles [31, 32] must be reconsidered. In this case, the rolling friction coefficient could depend on sliding friction, and new behavior models would need to be developed.

\section{References}

[1] J.K. Mitchell, K. Soga, Fundamentals of Soil Behavior (Wiley, New-York, 2005), ISBN 978-0-47146302-3

[2] K. Shinohara, M. Oida, B. Golman, Powder Technol. 107, 131 (2000)

[3] S. Torquato, Y. Jiao, Phys. Rev. E 80, 041104 (2009)

[4] E. Azéma, N. Estrada, F. Radjaï, Phys. Rev. E 86, 041301 (2012)

[5] H. Shin, J.C. Santamarina, J. Geotech. Geoenviron. Eng. 139, 353 (2013)

[6] S. Zhao, X. Zhou, Granul. Matter 19, 1 (2017)

[7] K.A. Murphy, A.K. MacKeith, L.K. Roth, H.M. Jaeger, Granul. Matter 21, 72 (2019)

[8] A. Donev, Science 303, 990 (2004)

[9] E. Azéma, F. Radjaï, Phys. Rev. E 81, 051304 (2010)

[10] D. Markauskas, R. Kačianauskas, A. Džiugys, R. Navakas, Granul. Matter 12, 107 (2010)

[11] T. Börzsönyi, B. Szabó, S. Wegner, K. Harth, J. Török, E. Somfai, T. Bien, R. Stannarius, Phys. Rev. E 86, 051304 (2012)

[12] E. Azéma, F. Radjaï, B. Saint-Cyr, J.Y. Delenne, P. Sornay, Phys. Rev. E 87, 052205 (2013)
[13] Y. Jiao, F.H. Stillinger, S. Torquato, Phys. Rev. E 81, 041304 (2010)

[14] A. Skinner, Geotechnique 19, 150 (1969)

[15] N. Abriak, Math. Comput. Model. 28, 121 (1998)

[16] L. Oger, S. Savage, D. Corriveau, M. Sayed, Mech. Mater. 27, 189 (1998)

[17] S.J. Antony, M.A. Sultan, Phys. Rev. E 75, 031307 (2007)

[18] N. Estrada, A. Taboada, F. Radjaï, Phys. Rev. E 78, 021301 (2008)

[19] X. Huang, K.J. Hanley, C. O'Sullivan, C.Y. Kwok, Int. J. Numer. Anal. Methods Geomech. 38, 1276 (2014)

[20] J. Gong, J. Zou, L. Zhao, L. Li, Z. Nie, Comput. Geotech. 113, 103105 (2019)

[21] K.M. Salerno, D.S. Bolintineanu, G.S. Grest, J.B. Lechman, S.J. Plimpton, I. Srivastava, L.E. Silbert, Phys. Rev. E 98, 050901 (2018)

[22] Z. Lu, A. Yao, A. Su, X. Ren, Q. Liu, S. Dong, Eng. Geol. 253, 36 (2019)

[23] Y. Zhu, Z. Nie, J. Gong, Particuology 52, 67 (2020)

[24] J.J. Moreau, Eur. J. Mech., A/Solids 13, 93 (1994)

[25] F. Dubois, V. Acary, M. Jean, Comptes Rendus Mécanique 346, 247 (2018)

[26] M. Renouf, F. Dubois, P. Alart, J. Comput. Appl. Math. 168, 375 (2004)

[27] J.J. Moreau, in Micromechanics of Granular Materials, edited by B. Cambou, M. Jean, F. Radjai (ISTE, London, UK, 2009), pp. 51-100, ISBN 978-0-47061161-6 978-1-84821-075-2

[28] A. Taboada, N. Estrada, F. Radjaï, Phys. Rev. Lett. 97, 098302 (2006)

[29] N. Estrada, E. Azéma, F. Radjai, A. Taboada, Phys. Rev. E 84, 011306 (2011)

[30] T. Binaree, E. Azéma, N. Estrada, M. Renouf, I. Preechawuttipong, Phys. Rev. E 102, 022901 (2020)

[31] C.M. Wensrich, A. Katterfeld, D. Sugo, Granul. Matter 16, 327 (2014)

[32] Y. Wang, F. Alonso-Marroquin, S. Xue, J. Xie, Particuology 18, 35 (2015) 